\title{
Laser-flash-photolysis-spectroscopy: a nondestructive method?
}

\author{
Jenny Schneider, ${ }^{\star a}$ Konstantin Nikitin, ${ }^{\text {ab }}$ Ralf Dillert ${ }^{a}$ \\ and Detlef W. Bahnemann*ab
}

Received 19th September 2016, Accepted 12th October 2016

DOI: $10.1039 / c 6 f d 00193 a$

\begin{abstract}
Herein, we report the effect of the laser illumination during the diffuse-reflectance laserflash-photolysis measurements on the morphological and optical properties of $\mathrm{TiO}_{2}$ powders. A grey-blue coloration of the $\mathrm{TiO}_{2}$ nanoparticles has been observed after intense laser illumination. This is explained by the formation of nonreactive trapped electrons accompanied by the release of oxygen atoms from the $\mathrm{TiO}_{2}$ matrix as detected by means of UV-vis and EPR spectroscopy. Moreover, in the case of the pure anatase sample a phase transition of some $\mathrm{TiO}_{2}$ nanoparticles located in the inner region from anatase to rutile occurred. It is suggested that these structural changes in $\mathrm{TiO}_{2}$ are caused by an energy and charge transfer to the $\mathrm{TiO}_{2}$ lattice.
\end{abstract}

\section{Introduction}

During the last few years the demand for fundamental studies in photocatalysis became very high, especially for investigations on powdered photocatalysts due to their better comparability with the results obtained in photocatalytic tests. Laser flash photolysis spectroscopy is a widely used method to study such fundamental processes such as the formation of charge carriers and their subsequent rapid recombination and interfacial transfer kinetics. ${ }^{1-3}$ In flash photolysis experiments the laser pulse can initiate the formation of transient species, which is usually accompanied with a change of the original optical features of the studied material. This change is spectroscopically monitored applying a flash lamp by measuring the transmittance for transparent samples or the reflectance for opaque samples, respectively, before and after the laser excitation. The optical changes induced by laser excitation in semiconductors are generally attributed to the formation of free and trapped electrons as well as of trapped holes. It is presupposed that upon illumination with the intense laser pulse no irreversible structural changes of the studied sample occur.

${ }^{a}$ Leibniz University Hannover, Institute for Technical Chemistry, 30167 Hannover, Germany. E-mail: schneider@iftc.uni-hannover.de

${ }^{b}$ Saint-Petersburg State University, Laboratory "Photoactive Nanocomposite Materials", Saint-Petersburg, 198504 Russia 
$\mathrm{TiO}_{2}$ is one of the most studied photocatalysts. In the literature different laser flash photolysis studies are presented dealing with the reaction dynamics of the charge carriers photogenerated in colloidal $\mathrm{TiO}_{2}$ suspensions, ${ }^{4-7} \mathrm{TiO}_{2}$ films,${ }^{8-12}$ and in dry $\mathrm{TiO}_{2}$ powders. ${ }^{13-16}$ However, most of the published transient absorption signals detected in $\mathrm{TiO}_{2}$ do not decay to the initial value observed before the laser pulse but exhibit long-lived transients within the time scale of observation. ${ }^{4,5,7,9,17,18}$ Serpone et al. ${ }^{4}$ attributed this long-lived transient absorption to deeply trapped electrons formed via so-called Auger-processes. On the other hand, these irreversible changes of the optical properties might be due to irreversible alternations of the material's properties caused by changes of its stoichiometric composition or by phase transitions. However, until now no experimentally based explanation for the observed change of the optical properties of $\mathrm{TiO}_{2}$ during the laser flash photolysis measurements has been given. Hence, we investigated the laser-induced changes of optical and morphological properties of the well-known anatase $\mathrm{TiO}_{2}$ photocatalyst Hombikat $\mathrm{UV} 100 \mathrm{TiO}_{2}$ by means of Raman, EPR, and UV-vis spectroscopy.

\section{Experimental}

Nanosecond diffuse reflectance laser flash photolysis spectroscopy measurements were performed using a set-up as reported in our previous paper. ${ }^{19}$ Briefly, the excitation of the sample proceeds with an excimer laser (LPX 200) with a wavelength of $351 \mathrm{~nm}$, and with a pulse duration of $15 \mathrm{~ns}$. The laser intensity per pulse varied between $7 \mathrm{~mJ} \mathrm{~cm}^{-2}$ per pulse and $60 \mathrm{~mJ} \mathrm{~cm}^{-2}$ per pulse. Powder in a flat quartz cuvette has been used for all experiments. Herewith, the illumination area of the laser beam and of the analyzing light are $0.5 \mathrm{~cm}^{2}$ and $0.196 \mathrm{~cm}^{2}$, respectively.

Raman spectra and micrographs were recorded with a SENTERRA Raman microscope. The spectral resolution of the analyzing spectrograph was $0.5 \mathrm{~cm}^{-1}$. A $532 \mathrm{~nm}$ laser was used as the excitation source, the power of the laser at the sample was $2.0 \mathrm{~mW}$. The presented spectra were obtained at room temperature by averaging five spectra with an integration time of $10 \mathrm{~s}$.

UV/vis diffuse reflectance spectra of the $\mathrm{TiO}_{2}$ powders were recorded on a Varian Cary 100 Scan UV-vis spectrophotometer equipped with a labsphere diffuse reflectance accessory. The reflectance data were converted to $F(R)$ values according to the Kubelka-Munk theory.

EPR spectra were registered with a MiniScope MS 400 X-band EPR spectrometer at liquid nitrogen temperature. The device parameters during the measurements were as follows: microwave frequency $9.42 \mathrm{GHz}$, microwave power $5 \mathrm{~mW}$, modulation frequency $100 \mathrm{kHz}$, modulation amplitude $0.15 \mathrm{mT}$.

\section{Materials}

Anatase $\mathrm{TiO}_{2}$ Hombikat UV100 provided by Huntsman, and rutile $\mathrm{TiO}_{2}$ provided by Crystal Global.

\section{Results}

Illumination of the $\mathrm{TiO}_{2}$ samples studied here by intense laser pulses leads to colour changes from white to grey-blue. This effect was stable and thus no further 
changes were observed under ambient conditions during few months. Hence, for a better understanding of the processes occurring at the $\mathrm{TiO}_{2}$ surface during the laser flash photolysis experiment it was important to characterize the samples before and after laser excitation. For this purpose the Hombikat UV100 powder has been analyzed by means of different spectroscopic methods presented below. To quantitatively and qualitatively understand the effect of the laser illumination the $\mathrm{TiO}_{2}$ sample has been exposed to a higher laser intensity than that usually employed, in a typical laser flash experiment, per pulse $\left(10 \times 50 \mathrm{~mJ} \mathrm{~cm}^{-2}\right.$ per pulse). Before its exposure to the pulsed laser irradiation the surface of the $\mathrm{TiO}_{2}$ (UV100) sample appeared entirely white. From the micrograph of the laser illuminated $\mathrm{TiO}_{2}$ surface, shown in Fig. 1(a), a nonhomogeneous distribution of colour centers is obvious.

Some areas exhibit dark coloration, while the majority of the sample's surface areas remained white. The Raman spectra of different sample areas are presented in Fig. 1(b). When the analyzing beam is focused on a white area, the Raman spectrum shows only anatase peaks: $149,200,395.5,513$ and $636.5 \mathrm{~cm}^{-1}$, which can be assigned to major modes of the anatase phase as $E_{g}, E_{g}, B_{1 g}$, and superpositions of $\mathrm{A}_{1 \mathrm{~g}}$ and $\mathrm{B}_{1 \mathrm{~g}}$, and $\mathrm{E}_{\mathrm{g}}$, respectively. ${ }^{20-23}$ The Raman spectrum of the dark areas exhibits additional peaks with maxima at $238,447.5$ and $610 \mathrm{~cm}^{-1}$. These three bands can be attributed to the two-phonon scattering, $\mathrm{E}_{\mathrm{g}}$, and $\mathrm{A}_{1 \mathrm{~g}}$ modes, of the rutile phase, respectively. ${ }^{21-24}$ Moreover, the highest anatase peak at $149 \mathrm{~cm}^{-1}$

(a)

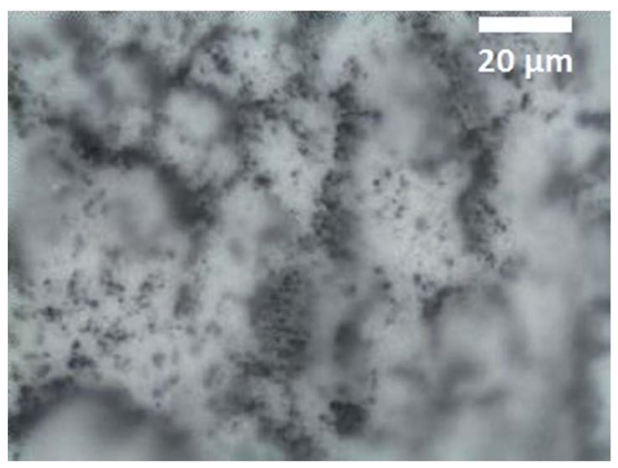

(b)

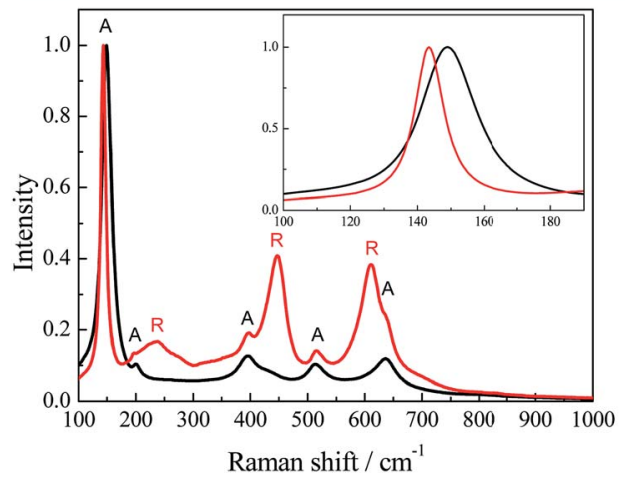

Fig. 1 (a) Micrograph of an illuminated UV100 sample surface (50× magnification). (b) Normalized Raman spectra of the illuminated UV100 sample: (black) white area and (red) dark area (exposed to the laser beam with a laser intensity of $10 \times 50 \mathrm{~mJ} \mathrm{~cm}^{-2}$ per pulse). 
has narrowed and shifted to $143.5 \mathrm{~cm}^{-1}$. Probably, such alteration happened due to an increase in particle size,$^{20}$ while the influence of the $\mathrm{B}_{1 \mathrm{~g}}$ mode of the rutile phase with a maximum at $143 \mathrm{~cm}^{-1}$ could be excluded for this interpretation because of its low line intensity. ${ }^{21-23}$

However, the detected anatase to rutile phase transition does not explain the dark grey-blue coloration of the $\mathrm{UV} 100 \mathrm{TiO}_{2}$ powder after the exposure to the laser. Fig. 2(a) presents the diffuse reflectance UV-vis spectra of untreated and of illuminated $\mathrm{TiO}_{2}$ powder, respectively. It can be seen that the absorption of the illuminated $\mathrm{TiO}_{2}$ in the visible range increases continuously with the wavelength. This very broad visible absorption spectrum indicates the presence of long-lived $\mathrm{Ti}^{3+}$ species in the $\mathrm{TiO}_{2}$ formed after the laser illumination. ${ }^{3}$ Apparently, these trap states are energetically distributed through the entire band gap exhibiting metallic-like character. Zhu et $a .^{25}$ reported a wide distribution of the trapped states for electrons within the entire bandgap. The presence of the $\mathrm{Ti}^{3+}$ centers in the laser treated $\mathrm{TiO}_{2}$ sample could furthermore be confirmed by means of electron paramagnetic resonance (EPR) spectroscopy. The initial uncolored sample does not show any EPR signal without UV-vis illumination, while the laser illuminated sample exhibits an intense signal as shown in Fig. 2(b). A broad peak with $g_{\text {iso }}=1.95$ with slight asymmetry can be assigned to $\mathrm{Ti}^{3+}$ centers in $\mathrm{TiO}_{2}{ }^{26,27}$

(a)

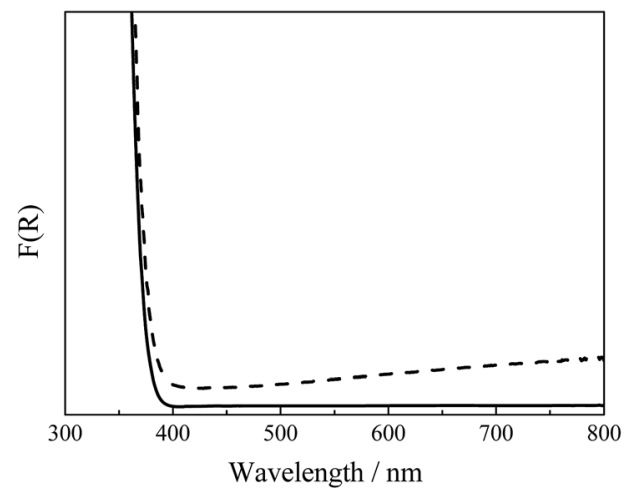

(b)

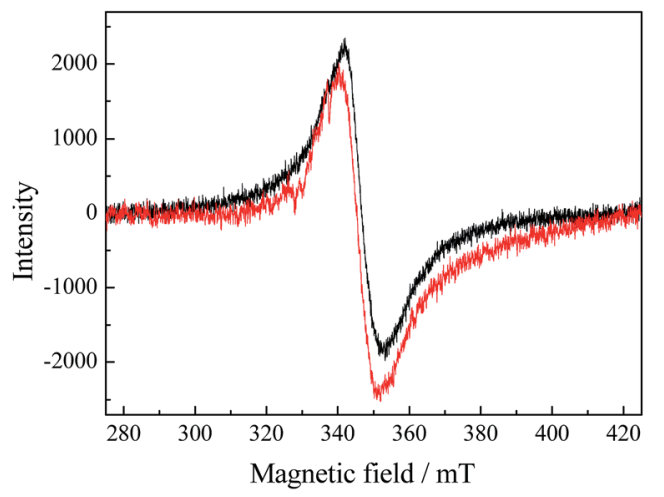

Fig. 2 (a) UV-vis diffuse reflectance spectra of (solid) untreated and (dash) illuminated UV100. (b) EPR spectra measured at $77 \mathrm{~K}$ of (black) illuminated $\mathrm{UV}_{100} \mathrm{TiO}_{2}$ anatase and (red) illuminated $\mathrm{R} 15 \mathrm{TiO}_{2}$ rutile (exposed to the laser beam with laser intensity: $10 \times$ $50 \mathrm{~mJ} \mathrm{~cm}{ }^{-2}$ per pulse). 
Most commonly, $\mathrm{Ti}^{3+}$ centers have axial symmetry and show specific signals which correspond to this anisotropy. ${ }^{26-29}$ In our case the axial signals are not resolved because the sample temperature is still too high for this and resolved signals could only be obtained at liquid helium temperature. ${ }^{26}$ Usually, similar EPR signals are assigned to $\mathrm{Ti}^{3+}$ in the rutile phase while anatase shows narrower $\mathrm{Ti}^{3+}$ signals with a higher $g$-value (1.98-1.99). ${ }^{27-29}$ The formation of $\mathrm{Ti}^{3+}$ species in the laser-generated rutile phase could be confirmed by recording the EPR-spectra of the laser illuminated rutile phase, which exhibits the same EPR signal as laser illuminated anatase $\mathrm{TiO}_{2}$ (see Fig. 2(b)). This also corresponds to the fact that rutile can be reduced much more easily than anatase.

Moreover, it should be noted that the $\mathrm{Ti}^{3+}$ centers detected here are stable in the presence of molecular oxygen as has been shown directly for $\mathrm{Ti}^{3+}$ centers obtained in black $\mathrm{TiO}_{2}$ consisting of mixed anatase/rutile phases. ${ }^{30}$ Hence, both the UV-vis spectra and the EPR results reveal that upon the laser excitation anatase/rutile $\mathrm{TiO}_{2}$ is formed exhibiting properties similar to those of black $\mathrm{TiO}_{2}$.

\section{Discussion}

As the Raman spectra (see Fig. 1(b)) revealed the excitation of the $\mathrm{UV} 100 \mathrm{TiO}_{2}$ anatase powder by a $351 \mathrm{~nm}$ laser pulse leads to an anatase to rutile phase transition in some parts of the material. Moreover, the formation of long-lived $\mathrm{Ti}^{3+}$ species located in the rutile phase is detected by means of EPR- and UV-visspectroscopy. These results clearly prove a partial laser-induced rearrangement of the $\mathrm{TiO}_{2}$ lattice. It is more likely that the reconstruction of the $\mathrm{TiO}_{2}$ structure occurs at the particle surfaces rather than in the bulk, since the former exhibits a higher defect density and thus a higher reactivity. ${ }^{31}$ However, the question arises whether the absorbed laser energy is invested into a temperature rise followed by a thermal anatase to rutile transition or whether it is directly transferred to the lattice inducing a bond breakage on the molecular level.

The laser-induced anatase to rutile phase transition has been already studied by several research groups, whereas some of them reported photoinduced thermal driven phase transitions. ${ }^{32,33}$ Wilkinson and Willsher pointed out that materials exhibiting large optical absorption coefficients such as $\mathrm{TiO}_{2}$ or $\mathrm{Fe}_{3} \mathrm{O}_{4}$ (i.e., with $\alpha>10^{4} \mathrm{~cm}^{-1}$ ) are likely to experience photoinduced thermal heating. ${ }^{34}$ Hereby the heat is homogeneously distributed over the illuminated volume resulting in a large temperature rise following the pulsed laser excitation. The temperature induced in $\mathrm{TiO}_{2}$ by a single laser pulse can be estimated according to the following equation: ${ }^{34}$

$$
Q_{0}=\frac{E_{0} \alpha}{\rho C}
$$

$Q_{0}$ : temperature $(\mathrm{K}), E_{0}$ : incident laser intensity per unit area $\left(\mathrm{J} \mathrm{cm}^{-2}\right.$ per pulse), $C$ : specific heat capacity $\left(\mathrm{J} \mathrm{g}^{-1} \mathrm{~K}^{-1}\right), \rho$ : density $\left(\mathrm{g} \mathrm{cm}^{-3}\right), \alpha$ : absorption coefficient $\left(\mathrm{cm}^{-1}\right)$.

Accordingly, the required temperature for the anatase to rutile phase transition of $680{ }^{\circ} \mathrm{C}$ can be achieved with a laser intensity of $40 \mathrm{~mJ} \mathrm{~cm} \mathrm{~cm}^{-2}$ per pulse (using values for the anatase phase), i.e., $\alpha=1.2 \times 10^{4} \mathrm{~cm}^{-1}, C=0.69 \mathrm{~J} \mathrm{~g}^{-1} \mathrm{~K}^{-1}$, and $\rho=1 \mathrm{~g} \mathrm{~cm}^{-3}$ (lower density value than that reported for bulk $\mathrm{TiO}_{2}\left(3.9 \mathrm{~g} \mathrm{~cm}^{-3}\right)$ due to the powdered samples used in the experiments). ${ }^{35}$ 
However, such a photoinduced thermal heating of the illuminated volume followed by a phase transition is less probable. It contradicts the studies of Stopper and Dohrmann, who demonstrated by means of time-resolved optoacoustic calorimetry that $88 \%$ of the released heat is dissipated in a few nanoseconds over the whole system, thus no significant temperature increase occurs. ${ }^{36}$ Recently, Ricci et al..$^{37,38}$ have shown by careful analysis of the Stokes to anti-Stokes Raman peak ratio that the local temperature of the nanoparticles during the laser illumination was $370 \mathrm{~K}$, thus a solely thermally driven anatase to rutile phase transition could be ruled out. Moreover, Yates et $a .^{39}$ have found that the photoinduced reconstruction of the $\mathrm{TiO}_{2}$ surface was not affected by cooling the surface below $150 \mathrm{~K}$.

In fact, the photoinduced desorption/adsorption of oxygen molecules on the surface of nanometer sized $\mathrm{TiO}_{2}$ particles is reported to play a fundamental role for the non-thermal phase transition induced upon laser illumination..$^{37,38,40,41}$ Hereby, the phase transition was attributed to surface modifications resulting from a proper depletion of adsorbed oxygen on the crystal surface. The desorption of oxygen molecules as well as atoms from the $\mathrm{TiO}_{2}$ lattice yielding oxygen vacancies leads to an enhancement of the surface chemical reactivity. Subsequently, stable chemical bonds are formed between the surfaces of neighboring anatase particles resulting in the formation of polycrystalline aggregates. The interface between the bound anatase particles, which are only present in the inner region of these agglomerated particles, provides the nucleation sites of the rutile phase. ${ }^{42}$ The formation of the rutile phase inside of such aggregates is in agreement with our results. We, furthermore, detected long-lived trapped electrons in the rutile phase, the lower reactivity of which evinces that they are located inside the $\mathrm{TiO}_{2}$ aggregate or cluster. The appearance of such non-reactive subsurface trapped electrons by tempering has previously been reported by Diebold et al. ${ }^{\mathbf{4 3}}$ The relative number of preferential nucleation sites for the anatase to rutile phase transition is found to increase as the population of oxygen vacancies at the particle surface increases. ${ }^{37,38}$ Zhou et $a .^{31,44}$ reported that the rutile phase nucleates exactly at the twin boundaries generated by anatase (112) surfaces. If the direct contact between anatase particles is prevented, the phase transformation was found to be retarded or prohibited at all. For example, no phase transformation has been observed for anatase $\mathrm{TiO}_{2}$ particles functionalized with nitric groups or with a $\mathrm{La}_{2} \mathrm{O}_{3}$ shell. ${ }^{37,42}$

The long-lived $\mathrm{Ti}^{3+}$ centers in $\mathrm{TiO}_{2}$ detected in the present study are most likely produced by oxygen removal during the laser illumination of anatase $\mathrm{TiO}_{2}$ (this has also been detected for the rutile phase). Desorption of one oxygen molecule leaves four additional electrons in the lattice. These electrons can either stay in the vacancy forming so-called $F$-centers, or they are transferred to $\mathrm{Ti}^{4+}$ yielding $\mathrm{Ti}^{3+}$ species. The conversion of $\mathrm{Ti}^{4+}$ to $\mathrm{Ti}^{3+}$ upon laser excitation at 355 $\mathrm{nm}$ has already been reported by Forsgren et $a .^{45}$ Concomitantly, the hydrophilicity of the surface was improved. Since we were able to detect such $\mathrm{Ti}^{3+}$ centers by means of EPR and UV-vis spectroscopy (see Fig. 2), this evinces that the phase transition observed in the present study occurs via a photoinduced oxygen desorption. However, the question arises, how the formation of the oxygen vacancies or of the rather long-lived $\mathrm{Ti}^{3+}$ species proceeds.

For example, Serpone et al. ${ }^{\mathbf{4 , 4 6}}$ reported that during laser flash photolysis studies long-lived $\mathrm{Ti}^{3+}$ centers can be produced via the so-called Auger 
recombination process. This Auger process is found to be the predominant mechanism when the number of photons exceeds the number of absorbing particles by far, which is in agreement with our experimental conditions. ${ }^{47,48}$ In the Auger process the energy released by the recombination of an electron-hole pair is absorbed by another electron (or hole) resulting in the formation of highly energetic electrons (or holes), which can dissipate the energy either by an electron ejection from the semiconductor nanoparticle resulting in the formation of solvated electrons or by a phonon emission leading to the generation of long-lived deeply trapped electrons. The presence of solvated electrons can be ruled out here since the experiments have been performed employing dry $\mathrm{TiO}_{2}$ powders instead of aqueous $\mathrm{TiO}_{2}$ suspensions. However, the only experimental evidence for the formation of such $\mathrm{Ti}^{3+}$ species via an Auger process is the fact that the published transient absorption signals detected in $\mathrm{TiO}_{2}$ do not decay to the initial value observed before the laser pulse but exhibit a long-lived transient absorption

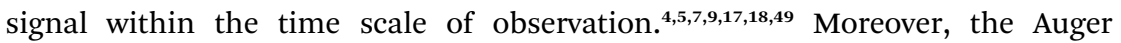
recombination is normally observed in heavily doped, direct bandgap semiconductors. In direct bandgap semiconductors the recombination proceeds upon releasing photons, while in indirect semiconductors in most cases phonons are released, the energy of which is usually not high enough to excite other charge carriers. ${ }^{50}$

Lisachenko et al. ${ }^{51}$ proposed two possible processes to explain for the photodesorption of oxygen molecules from the metal oxide surface:

(1) Photothermic processes, whereby the excitation energy is transformed into phonon energy of the solid resulting in the excitation of the vibrational mode of the surface-atom bond followed by the rupture of this bond.

(2) Photoelectronic processes, whereby the excitation energy is absorbed by the atom-surface bond thus changing the potential energy of the bond directing it towards the repulsive branch of the potential curve for the atom-surface interaction. Subsequently, the potential energy is converted into kinetic energy of the emitted particles, thus entailing the nature of the oxygen desorption. This mechanism is based on the research of Terenin, who pointed out that the emitted particle can gain the rest of the photon energy, provided that the latter exceeds the energy necessary for bond breaking. ${ }^{52}$

Based on Time-of-Flight (TOF) spectroscopy measurements, which allow the determination of the temperature of the desorbed molecules, Lisachenko ruled out the photothermic model. ${ }^{51}$ In photothermic processes the average velocity of the desorbed molecules should correspond to the surface temperature. The detected kinetic temperature of the desorbed oxygen molecules, however, exceeds by far the highest surface temperature produced by the laser beam. The photodesorption of oxygen from the $\mathrm{TiO}_{2}$ surface is therefore rather found to proceed via photoelectronic processes induced by electronic transitions. Hereby, the photodesorption of preadsorbed oxygen molecules (which are present on the metal oxide surface is a partially reduced form, i.e., as superoxide radical anions) is caused by the capture of a photogenerated hole: ${ }^{53-55}$

$$
\mathrm{h}_{\mathrm{VB}}{ }^{+}+\mathrm{O}_{2}^{-}{ }^{-} \text {(ads) } \rightarrow \mathrm{O}_{2}(\mathrm{~g}) \uparrow
$$

The desorption of the oxygen molecule from the $\mathrm{TiO}_{2}$ surface lattice includes the partial neutralization of the oxygen anion: ${ }^{39}$ 


$$
\mathrm{O}^{2-}+\mathrm{h}^{+} \rightarrow \mathrm{O}^{-}
$$

The oxidation of further oxygen anions results in the transformation of two neighboring $\mathrm{O}^{2-}$ anions into molecular oxygen. Accordingly, two single-photon excitations are required for the formation of one oxygen vacancy:

$$
2 h \nu+2 \mathrm{O}_{\mathrm{s}}^{-} \rightarrow \mathrm{O}_{2} \uparrow+2 \mathrm{e}^{-}
$$

The structural changes of the $\mathrm{TiO}_{2}$ lattice observed in the present study can only be induced by the removal of strongly bound species. The photooxidation of lattice bound oxygen via photogenerated holes resulting in the release of molecular oxygen has been proposed as a mechanism for $\mathrm{O}_{2}$ formation by Salvador $^{56}$ and for the gas phase photocatalytic oxidation of organic compounds by Pichat $^{57,58}$ and Lisachenko. ${ }^{59}$

The idea behind the mechanism of photoelectronic processes including the excitation energy transfer to the surface-atom bond resulting in the formation of an oxygen vacancy is also supported by other experimental techniques such as ion and electron bombardment, where the kinetic energy of the accelerated ions is transferred to the $\mathrm{TiO}_{2}$ lattice. ${ }^{60,61}$ Actually, Fukushima et al. ${ }^{62}$ observed the anatase to rutile transition during the $\mathrm{TiO}_{2}$ film formation by means of ion bombardment. Moreover, the laser induced phase transition of Silicon has been explained by energy transfer from an optically excited electron-hole plasma to the crystal lattice resulting in the crystal melting. This process is known as nonthermal melting. ${ }^{63-65}$ However, the latter occurs only upon femtosecond excitation at a laser fluency of about $0.2 \mathrm{~J} \mathrm{~cm}^{-2}$.

According to the above presented discussion, in the photoinduced processes studied here the absorbed light energy is transferred to the lattice inducing bondbreaking and displacement of atoms involved in the reconstructive transformation from anatase to rutile. According to Stopper and Dohrmann, 88\% of the energy absorbed by colloidal $\mathrm{TiO}_{2}$ particles after the laser pulse is dissipated in less than one nanosecond as heat over the whole system as a consequence of the charge-carrier recombination, while the remaining $12 \%$ can be stored for at least $2 \mu \mathrm{s} .{ }^{36}$ Applying a laser pulse with an excitation wavelength of $351 \mathrm{~nm}$, a total energy of $347 \mathrm{~kJ} \mathrm{~mol}^{-1}$ can be released as heat. According to Stopper and Dohrmann, $305 \mathrm{~kJ} \mathrm{~mol}^{-1}$ will be dissipated over the whole particulate system, while 42 $\mathrm{kJ} \mathrm{mol}^{-1}$ can be utilized locally. Employing a laser intensity of $23 \mathrm{~mJ} \mathrm{~cm}^{-2}$ per pulse, one UV100 anatase nanoparticle can absorb 522 photons as estimated according to eqn (5):

$$
N_{\mathrm{p}}=\frac{E_{0} \lambda_{\mathrm{exc}} 1 / 6 \pi d^{3} \alpha}{c h}
$$

$c$ : speed of light $\left(\mathrm{m} \mathrm{s}^{-1}\right), h$ : Planck's constant ( $\left.\mathrm{J}\right)$.

Assuming that every absorbed photon is utilized as much as $21924 \mathrm{~kJ} \mathrm{~mol}^{-1}$ can be locally converted into bond breaking. For the cleavage of one Ti-O bond an energy of $315 \mathrm{~kJ} \mathrm{~mol}^{-1}$ is required. Hence, with one laser pulse of $23 \mathrm{~mJ} \mathrm{~cm}^{-2}$, up to $70 \mathrm{Ti}-\mathrm{O}$ bonds per $\mathrm{TiO}_{2}$ anatase particle can be broken. For the transition of bulk anatase to rutile it was reported that 7 out of the $24 \mathrm{Ti}-\mathrm{O}$ bonds per unit cell need to be broken leading to the cooperative displacement of both Ti and $\mathrm{O} \cdot{ }^{37,41} \mathrm{It}$ is more likely, however, that most of the $\mathrm{Ti}-\mathrm{O}$ bonds are broken at the $\mathrm{TiO}_{2}$ 
surface. The anatase nanoparticles of UV100 are spherical in shape, therefore the number of $\mathrm{TiO}_{2}$ unit cells present at the surface is estimated to be 180 . Hence, during each laser pulse $3 \mathrm{Ti}-\mathrm{O}$ bonds per unit cell can be broken. Subsequently, after the second laser pulse, the phase transition can be initiated.

The inhomogeneous distribution of the black spots containing the rutile phase and long-lived $\mathrm{Ti}^{3+}$-centers (see Fig. 1(a)) observed here for the laser irradiated powder samples indicates different reactivities of the $\mathrm{TiO}_{2}$ clusters at the surface. Assuming a homogeneous distribution of the photons over the illuminated $\mathrm{TiO}_{2}$ surface all exposed $\mathrm{TiO}_{2}$ units should be destabilized during the laser pulse. As a result bond breaking and atom displacement can occur. After the laser illumination the more stable $\mathrm{TiO}_{2}$ units manage to return back to the initial state (this self-repair mechanism can also be supported by photo adsorption of the oxygen or water molecules), thus the initial anatase phase remains (see Fig. 1(a)). However, the more reactive $\mathrm{TiO}_{2}$ clusters (most likely due to a higher defect density) undergo intense irreversible structural changes such as oxygen removal followed by the observed phase transition. The latter results in the reported grey-blue coloration of the $\mathrm{TiO}_{2}$ sample. As a matter of fact, the photodeposition of metal nanoparticles on metal oxide surfaces also results in an inhomogeneous distribution of metal islands on the surface. ${ }^{66}$

\section{Conclusions}

In the present study the irreversible changes of $\mathrm{TiO}_{2}$ during the diffuse reflectance laser flash photolysis experiments have been experimentally analyzed. The following laser-induced processes have been suggested to occur in anatase $\mathrm{TiO}_{2}$ powder upon illumination with intense laser pulses: formation of electron-hole pairs, followed by energy and charge transfer to the $\mathrm{TiO}_{2}$ lattice. The regions of the $\mathrm{TiO}_{2}$ surface with high defect density undergo irreversible changes, such as removal of the lattice oxygen resulting in the formation of oxygen vacancies. This enhances the surface reactivity of the $\mathrm{TiO}_{2}$ particles, thus at the interfaces of the anatase nanoparticles a phase transition to rutile occurs. The presence of $\mathrm{Ti}^{3+}$ centers in the illuminated powder evinces that the phase transition to rutile occurs via an oxygen release from the $\mathrm{TiO}_{2}$ surface induced by the electron/energy transfer. However, the major part of the $\mathrm{TiO}_{2}$ nanoparticles are able to conduct self-repair processes and therefore do not exhibit any significant morphological and structural changes.

These findings clearly demonstrate that the obtained transient absorption signals could be strongly influenced by such irreversible changes of the material and need to be treated with caution. This will be the topic of subsequent publications.

Furthermore, the message of the study presented here so far is that upon illumination with intense pulsed laser light structural changes of the $\mathrm{TiO}_{2}$ photocatalyst may occur. These findings are essential for the evaluation of the underlying photocatalytic reactions. Although there are many studies which present such structural changes of the $\mathrm{TiO}_{2}$ nanoparticles upon illumination, ${ }^{32,57,58,67-71}$ this effect has rarely been considered for the interpretation of the photoinduced photocatalytic processes. ${ }^{72}$ Based upon these possible reactions it is certainly highly advisable to study the role of the surface reorganization on the reactions induced upon illumination. The knowledge of these processes can most probably open new design pathways for future photocatalysts. 
This work was funded in part by the German Federal Ministry of Education and Research (contract no. 13N13350, "PureBau - Untersuchung von Werkstoffsystemen für photokatalytisch hocheffiziente Baustoffe-Teilvorhaben: Oberflächenchemie der Photokatalysatoren und der Werkstoffe") and by the Project "Establishment of the Laboratory Photoactive Nanocomposite Materials" no. 14.Z50.31.0016 supported by a Mega-grant of the Government of the Russian Federation. This work was supported by the Global Research Laboratory (GRL) Programme (NRF-2014K1A1A2041044) funded by the Korea government (MSIP) through NSF. The authors thank Jinlin Nie for providing the EPR spectra.

\section{References}

1 P. V. Kamat, Prog. React. Kinet., 1994, 19, 277.

2 H. H. Mohamed, R. Dillert and D. W. Bahnemann, Chem.-Eur. J., 2012, 18, 4314.

3 J. Schneider, M. Matsuoka, M. Takeuchi, J. Zhang, Y. Horiuchi, M. Anpo and D. W. Bahnemann, Chem. Rev., 2014, 114, 9919.

4 N. Serpone, D. Lawless, R. Khairutdinov and E. Pelizzetti, J. Phys. Chem., 1995, 99, 16655.

5 D. Bahnemann, A. Henglein, J. Lilie and L. Spanhel, J. Phys. Chem., 1984, 88, 709.

6 M. Grätzel and A. J. Frank, J. Phys. Chem., 1982, 86, 2964.

7 I. A. Shkrob and M. C. Sauer, J. Phys. Chem. B, 2004, 108, 12497.

8 S. A. Haque, Y. Tachibana, R. L. Willis, J. E. Moser, M. Grätzel, D. R. Klug and J. R. Durrant, J. Phys. Chem. B, 2000, 104, 538.

9 T. Yoshihara, R. Katoh, A. Furube, Y. Tamaki, M. Murai, K. Hara, S. Murata, H. Arakawa and M. Tachiya, J. Phys. Chem. B, 2004, 108, 3817.

10 T. Yoshihara, Y. Tamaki, A. Furube, M. Murai, K. Hara and R. Katoh, Chem. Phys. Lett., 2007, 438, 268.

11 Y. Tamaki, A. Furube, R. Katoh, M. Murai, K. Hara, H. Arakawa and M. Tachiya, C. R. Chim., 2006, 9, 268.

12 Y. Tamaki, K. Hara, R. Katoh, M. Tachiya and A. Furube, J. Phys. Chem. C, 2009, 113, 11741.

13 C. J. Willsher, J. Photochem., 1985, 28, 229.

14 R. W. Kessler, G. Krabichler, S. Uhl, D. Oelkrug, W. P. Hagan, J. Hyslop and F. Wilkinson, Opt. Acta Int. J. Opt., 1983, 30, 1099.

15 A. Yamakata, J. J. M. Vequizo and H. Matsunaga, J. Phys. Chem. C, 2015, 119, 24538.

16 A. Kafizas, X. Wang, S. R. Pendlebury, P. Barnes, M. Ling, C. Sotelo-Vazquez, R. Quesada-Cabrera, C. Li, I. P. Parkin and J. R. Durrant, J. Phys. Chem. A, 2016, 120, 715.

17 G. Rothenberger, J. Moser, M. Gratzel, N. Serpone and D. K. Sharma, J. Am. Chem. Soc., 1985, 107, 8054.

18 D. W. Bahnemann, M. Hilgendorff and R. Memming, J. Phys. Chem. B, 1997, 101, 4265.

19 J. Schneider, K. Nikitin, M. Wark, D. W. Bahnemann and R. Marschall, Phys. Chem. Chem. Phys., 2016, 18, 10719. 
20 W. F. Zhang, Y. L. He, M. S. Zhang, Z. Yin and Q. Chen, J. Phys. D: Appl. Phys., 2000, 33, 912.

21 M.-S. Zhang, Z. Yin, Q. Chen, W. Xijun and J. Xiaoli, Ferroelectrics, 1995, 168, 131.

22 J. Parker and R. Siegel, J. Mater. Res., 1990, 5, 1246.

23 U. Balachandran and N. Eror, J. Solid State Chem., 1982, 42, 276.

24 J. Parker and R. Siegel, Appl. Phys. Lett., 1990, 57, 943.

25 M. Zhu, Y. Mi, G. Zhu, D. Li, Y. Wang and Y. Weng, J. Phys. Chem. C, 2013, 117, 18863.

26 V. Khomenko, K. Langer, H. Rager and A. Fett, Phys. Chem. Miner., 1998, 25, 338.

27 R. F. Howe and M. Grätzel, J. Phys. Chem., 1985, 89, 4495.

28 T. Berger, M. Sterrer, O. Diwald and E. Knözinger, ChemPhysChem, 2005, 6, 2104.

29 J. B. Priebe, J. R. Radnik, A. J. Lennox, M.-M. Pohl, M. Karnahl, D. Hollmann, K. Grabow, U. Bentrup, H. Junge and M. Beller, ACS Catal., 2015, 5, 2137.

30 A. Naldoni, M. Allieta, S. Santangelo, M. Marelli, F. Fabbri, S. Cappelli, C. L. Bianchi, R. Psaro and V. Dal Santo, J. Am. Chem. Soc., 2012, 134, 7600.

31 Y. Zhou and K. A. Fichthorn, J. Phys. Chem. C, 2012, 116, 8314.

32 H. Y. Lee, W. L. Lan, T. Y. Tseng, D. Hsu, Y. M. Chang and J. G. Lin, Nanotechnology, 2009, 20, 315702.

33 H. L. Ma, J. Y. Yang, Y. Dai, Y. B. Zhang, B. Lu and G. H. Ma, Appl. Surf. Sci., 2007, 253, 7497.

34 F. Wilkinson, C. J. Willsher, S. Uhl, W. Honnen and D. Oelkrug, J. Photochem., 1986, 33, 273.

35 D. A. H. Hanaor and C. C. Sorrell, J. Mater. Sci., 2011, 46, 855.

36 K. Stopper and J. K. Dohrmann, Z. Phys. Chem., 2000, 214, 555.

37 P. C. Ricci, C. M. Carbonaro, L. Stagi, M. Salis, A. Casu, S. Enzo and F. Delogu, J. Phys. Chem. C, 2013, 117, 7850.

38 P. C. Ricci, A. Casu, M. Salis, R. Corpino and A. Anedda, J. Phys. Chem. C, 2010, 114, 14441.

39 S. Mezhenny, P. Maksymovych, T. L. Thompson, O. Diwald, D. Stahl, S. D. Walck and J. T. Yates Jr, Chem. Phys. Lett., 2003, 369, 152.

40 L. Stagi, C. M. Carbonaro, R. Corpino, D. Chiriu and P. C. Ricci, Phys. Status Solidi B, 2015, 252, 124.

41 G. C. Vasquez, M. A. Peche-Herrero, D. Maestre, A. Gianoncelli, J. RamarezCastellanos, A. Cremades, J. M. Gonzalez-Calbet and J. Piqueras, J. Phys. Chem. C, 2015, 119, 11965.

42 J. Zhang, M. Li, Z. Feng, J. Chen and C. Li, J. Phys. Chem. B, 2006, 110, 927.

43 U. Diebold, J. Lehman, T. Mahmoud, M. Kuhn, G. Leonardelli, W. Hebenstreit, M. Schmid and P. Varga, Surf. Sci., 1998, 411, 137.

44 R. L. Penn and J. F. Banfield, Am. Mineral., 1999, 84, 871.

45 J. Forsgren, M. D. Paz, B. Leon and H. Engqvist, J. Mater. Sci.: Mater. Med., 2013, 24, 11.

46 N. Serpone, D. Lawless and R. Khairutdinov, J. Phys. Chem., 1995, 99, 16646.

47 A. W. Henglein and H. Weller, Photochemical energy conversion, ed. J. R. Norris and D. Meisel, Elsevier, New York, 1989, p. 161.

48 A. K. Ghosh, F. G. Wakim and R. R. Addiss, Phys. Rev., 1969, 184, 979.

49 A. Y. Ahmed, T. A. Kandiel, T. Oekermann and D. Bahnemann, J. Phys. Chem. Lett., 2011, 2, 2461. 
50 F. J. Knorr, C. C. Mercado and J. L. McHale, J. Phys. Chem. C, 2008, 112, 12786.

51 A. A. Lisachenko, J. Photochem. Photobiol., A, 2008, 196, 127.

52 A. N. Terenin, Uchenye zapiski LGU (rus), 1939, 5, 26.

53 Z. Zhang and J. T. Yates, J. Phys. Chem. C, 2010, 114, 3098.

54 J. T. Yates, Surf. Sci., 2009, 603, 1605.

55 T. L. Thompson and J. T. Yates, J. Phys. Chem. B, 2005, 109, 18230.

56 P. Salvador, Prog. Surf. Sci., 2011, 86, 41.

57 H. Courbon, M. Formenti and P. Pichat, J. Phys. Chem., 1977, 81, 550.

58 H. Courbon and P. Pichat, C. R. Seances Acad. Sci., Ser. C, 1977, 285, 171.

59 V. V. Titov, R. V. Milchaylov and A. A. Lisachenko, J. Phys. Chem. C, 2014, 118, 21986.

60 J.-M. Pan, B. L. Maschhoff, U. Diebold and T. E. Madey, J. Vac. Sci. Technol., A, 1992, 10, 2470.

61 P. J. F. M. L. Knotek, Phys. Rev. Lett., 1978, 40, 964.

62 I. W. Boyd, E. Rimini, K. Fukushima and I. Yamada, Appl. Surf. Sci., 1989, 43, 32.

63 C. V. Shank, R. Yen and C. Hirlimann, Phys. Rev. Lett., 1983, 50, 454.

64 P. Stampfli and K. Bennemann, Phys. Rev. B: Condens. Matter Mater. Phys., 1994, 49, 7299.

65 T. Zier, E. S. Zijlstra, A. Kalitsov, I. Theodonis and M. E. Garcia, Struct. Dyn., 2015, 2, 054101.

66 D. Friedmann, H. Hansing and D. Bahnemann, Z. Phys. Chem., 2007, 221, 329.

67 S. Sato, T. Kadowaki and K. Yamaguti, J. Phys. Chem., 1984, 88, 2930.

68 A. L. A. Parussulo, M. F. G. Huila, K. Araki and H. E. Toma, Langmuir, 2011, 27, 9094.

69 C. Y. Wang, R. Pagel, J. K. Dohrmann and D. W. Bahnemann, C. R. Chim., 2006, 9, 761.

70 J. F. Montoya, D. W. Bahnemann, J. Peral and P. Salvador, ChemPhysChem, 2014, 15, 2311.

71 J. F. Montoya, I. Ivanova, R. Dillert, D. W. Bahnemann, P. Salvador and J. Peral, J. Phys. Chem. Lett., 2013, 4, 1415.

72 C. B. Mendive, D. Hansmann, T. Bredow and D. Bahnemann, J. Phys. Chem. C, 2011, 115, 19676. 